https://doi.org/10.23913/ride.v10i20.637

Artículos Científicos

\title{
Perfil TIC de estudiantes de la licenciatura en Educación de la UNACAR
}

\section{ICT Profile of Students of the UNACAR's Bachelor of Education}

Perfil de TIC dos alunos do Bacharelado em Educação da UNACAR

Gisela Aquilea Diez Irizar

Universidad Autónoma del Carmen, México gisela_diez@yahoo.com.mx https://orcid.org/0000-0002-8268-7170

Beatriz Herrera Sánchez Universidad Autónoma del Carmen, México bhsanchez70@hotmail.com https://orcid.org/0000-0002-6081-8985

Jesús Alejandro Flores Hernández Universidad Autónoma del Carmen, México jflores@pampano.unacar.mx https://orcid.org/0000-0002-5789-6990 


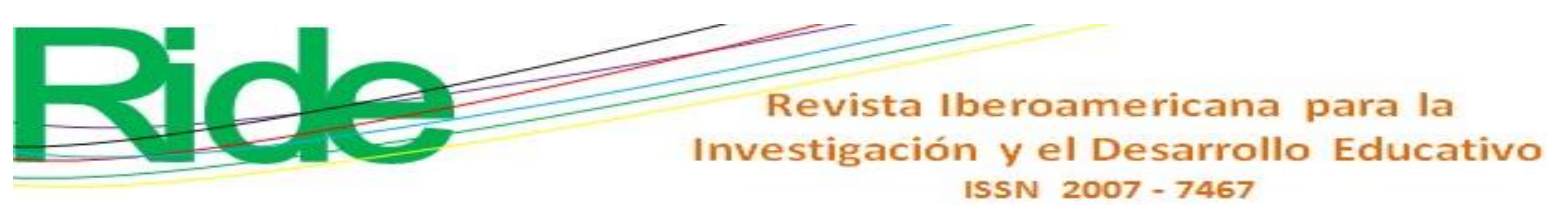

\section{Resumen}

El objetivo central de este texto es dar a conocer el perfil que tienen los estudiantes de primer semestre de la licenciatura en Educación de la Universidad Autónoma del Carmen (Unacar) en cuanto a habilidades, conocimientos y condiciones materiales individuales y colectivas para el empleo de las tecnologías de la información y la comunicación (TIC). Para ello, se realizó una investigación de tipo cuantitativa, con un alcance descriptivo. El instrumento, una encuesta con 20 reactivos de opción múltiple, estuvo basado en el Cuestionario sobre usos de las TIC entre estudiantes universitarios barineses (Universitat Oberta de Catalunya, 2006), el cual fue adaptado a las características de la población objeto de estudio, las condiciones particulares de las universidades en México, así como la zona en la que se realiza este estudio. Se seleccionó una muestra no aleatoria de 52 estudiantes de los dos grupos primer ingreso, en el ciclo escolar de febrero a julio del 2019, de la licenciatura en Educación de la Unacar, ubicada en el estado de Campeche. Los resultados obtenidos indican que, del total de la muestra, $44 \%$ permanece conectado a Internet de 13 a 20 horas semanales. Asimismo, $50 \%$ de los encuestados no posee equipo de cómputo personal en sus casas, por lo que realizan sus tareas en algún ciber (33\%) o en la universidad (25\%). A pesar de que $44 \%$ comenzó a utilizar la computadora entre los 3 y 10 años de edad, solo $19 \%$ la utiliza para actividades académicas; $75 \%$ emplea el Internet para comunicarse con sus compañeros de clase por correo electrónico, fundamentalmente. Aunado a ello, $46 \%$ no ha participado en foros en línea ni tiene experiencia en varios programas informáticos; $49 \%$ emplea el Internet solo para buscar información para las clases. A pesar de todo, $92 \%$ de los estudiantes consideran muy importante el empleo de las TIC en su futura profesión.

Palabras clave: competencias tecnológicas, difusión selectiva de información, enseñanza superior, tecnología educacional. 


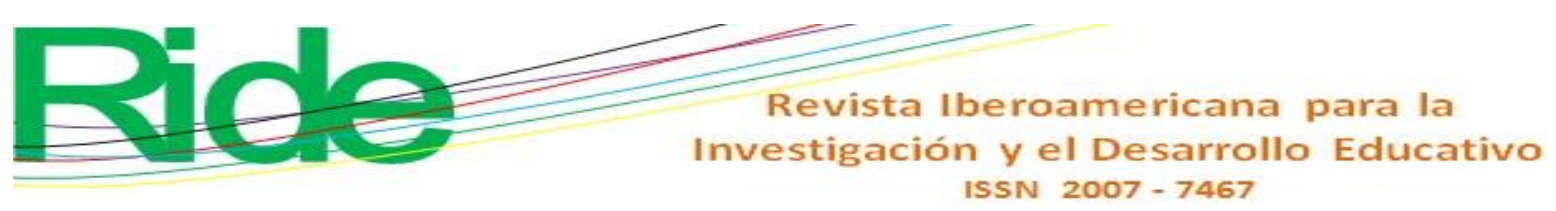

\section{Abstract}

The central objective of this text is to publicize the profile of first semester students of the Bachelor of Education degree of the Universidad Autonoma de Carmen (Unacar) about individual and collective skills, knowledge and material conditions for the use of information and communication technologies (TIC). For this, a quantitative research was carried out, with a descriptive scope. The instrument, a survey with 20 questions of multiple choice, was based on the Questionnaire about the uses of TIC among University students in Barcelona (Universitat Oberta de Catalunya, 2006), which was adapted to the characteristics of the population under study, the particular conditions of universities in Mexico, as well as the area where this study is performed, A non-random sample of 52 students of the first two admission groups from February to July 2019 was selected, of the education degree from UNACAR, located in the state of Campeche, The results obtained indicate that, of the total sample, 44\% remain connected to the Internet from 13 to 20 hours per week. Likewise, 50\% of the respondents do not have personal computer at home, so they carry out their homeworks in a internet cafe (33\%) or at the university (25\%). Despite the fact that $44 \%$ started using the computer between 3 and 10 years of age, only 19\% use it for academic activities; 75\% use the Internet to communicate with their classmates by email, fundamentally. In addition, $46 \%$ have not participated in online forums or have experience in various computer programs; $49 \%$ use the Internet only to search for information for classes. in spite of everything, 92\% of students consider the use of TIC very important in their profession future.

Keywords: technological competencies, selective information dissemination, higher education, educational technology. 


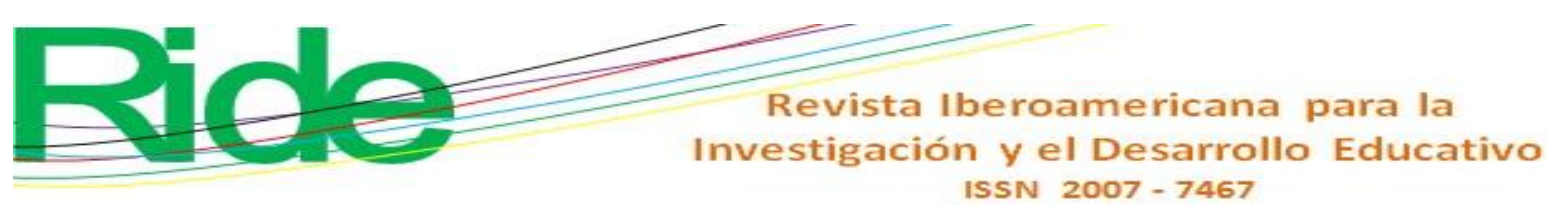

\section{Resumo}

O objetivo principal deste texto é divulgar o perfil dos alunos do primeiro semestre do Bacharelado em Educação da Universidade Autônoma de Carmen (Unacar) em termos de habilidades individuais e coletivas, conhecimentos e condições materiais para o emprego de tecnologias da informação e comunicação (TIC). Para isso, foi realizada uma pesquisa quantitativa, com escopo descritivo. O instrumento, uma pesquisa com 20 itens de múltipla escolha, baseou-se no Questionário sobre uso de TIC entre estudantes universitários de Barines (Universitat Oberta da Catalunya, 2006), adaptado às características da população estudada, o condições particulares das universidades do México, bem como a área em que este estudo é realizado. Uma amostra não aleatória de 52 alunos dos dois grupos de primeira admissão foi selecionada, no ano letivo de fevereiro a julho de 2019, pelo Bacharelado em Educação da Unacar, localizado no estado de Campeche. Os resultados obtidos indicam que, do total da amostra, 44\% permanecem conectados à Internet de 13 a 20 horas por semana. Da mesma forma, $50 \%$ dos entrevistados não possuem equipamento de computação pessoal em casa, portanto realizam suas tarefas em um ambiente cibernético (33\%) ou na universidade (25\%). Apesar de $44 \%$ começarem a usar o computador entre 3 e 10 anos, apenas 19\% o utilizam para atividades acadêmicas; $75 \%$ usam a Internet para se comunicar com seus colegas de classe por e-mail, fundamentalmente. Além disso, $46 \%$ não participaram de fóruns on-line ou possuem experiência em vários programas de computador; $49 \%$ usam a Internet apenas para procurar informações para as aulas. Apesar de tudo, 92\% dos estudantes consideram muito importante o uso das TIC em sua futura profissão.

Palavras-chave: competências tecnológicas, disseminação seletiva de informações, ensino superior, tecnologia educacional.

Fecha Recepción: Octubre 2019

Fecha Aceptación: Marzo 2020 


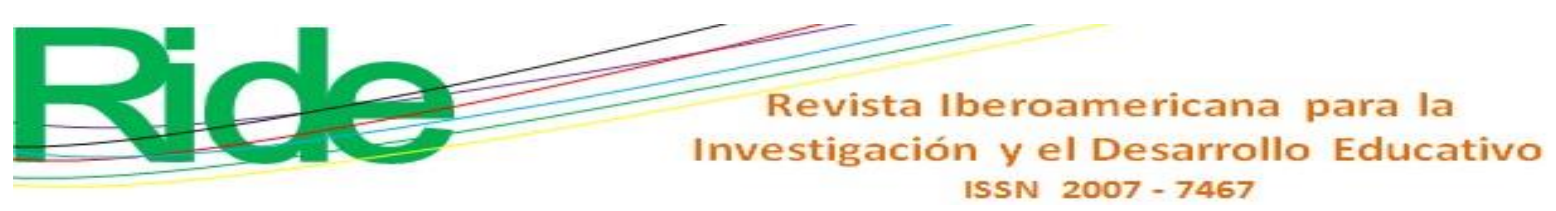

\section{Introducción}

La entrada de las tecnologías de la información y la comunicación (TIC) en la enseñanza superior actual plantea un cambio en el paradigma educativo, puesto que demanda de una formación acorde a la sociedad del conocimiento y de la información.

Este cambio afecta a la sociedad, a la individualidad y a la educación de diferentes maneras, ya que todo lo que nos rodea es tecnología. A decir de Arras, Torres y García (2011): "Las competencias en TIC han pasado a formar parte de los requisitos que se demandan en muchos de los puestos laborales" (p. 2). Por lo tanto, existe un compromiso por parte de las universidades de desarrollar estas competencias en todas las especialidades y que los estudiantes y docentes estén capacitados para la práctica de estas nuevas tecnologías en su día a día.

En esa línea, en los últimos años, las universidades han realizado innovaciones con el empleo de las tecnologías, y también han demostrado su interés en conocer cómo los estudiantes se apropian del manejo de las TIC para las actividades académicas.

De ello son evidencia algunas de las investigaciones realizadas de un tiempo para acá. Por ejemplo, la pesquisa de Cázares y Urbiola (2015) que involucra universidades públicas del Bajío de México: instituciones de San Luis de Potosí, Querétaro y Guanajuato, y que tuvo como objetivo indagar el implicamiento de las TIC en el ámbito educativo desde la perspectiva de los estudiantes. Como resultado, se obtuvo que los alumnos son solo consumidores de información, a pesar de tener las condiciones materiales y personales para activar el uso de la tecnología en la labor académica.

Posada (2015), por su parte, realizó un estudio en la Universidad de San Buenaventura, Cali, Colombia, con una muestra de 30 estudiantes, a quienes se les aplicó varios instrumentos, entrevistas, grupos focales y diarios de campo, entre otros, con la intención de conocer sus impresiones sobre la comunicación mediada por la tecnología. Entre los resultados, Posada (2015) destaca que los jóvenes reconocen la importancia del uso correcto de las tecnologías en la educación. Incluso se cuestionan, desde un punto de vista ético, el deber ser de las TIC en la sociedad actual.

La Universidad Autónoma del Carmen (Unacar), en el estado de Campeche, México, no es ajena a las demandas de la sociedad, a las preocupaciones globales en la enseñanza, la vida cotidiana y los empleadores, por lo que la estructura de su modelo educativo, el Modelo Educativo Acalán (Unacar, 2017), se basa en las competencias que debe desarrollar el 


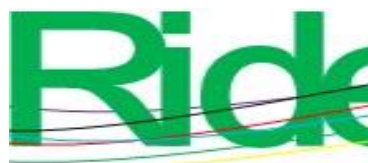

Revista Iberoamericana para la
Investigación y el Desarrollo Educativo
ISSN $2007-7467$

estudiante a lo largo de su trayectoria académica con el objetivo de cumplir con las demandas laborales del siglo XXI.

Los estudios acerca del enfoque por competencias se remontan al siglo pasado, cuando el término comenzó a ser familiar desde dos perspectivas diferentes (López, 2013): la psicología conductual de Skinner y la lingüística de Chomsky. El propio López (2013) considera que la competencia es "como un comportamiento efectivo, basado en la conducta observable efectiva y verificable de las personas" (p. 34). Más adelante este concepto se fue considerando y aplicando en distintos contextos; sin embargo, hay dos ámbitos en donde especialmente arraigó con fuerza, ambos familiares a nuestro entorno: el ámbito empresarial y el formativo.

En ambos contextos (empresarial y educativo) las competencias son dominantes. Por un lado, en las empresas se exige que el profesionista esté capacitado para realizar las tareas que demanda su área, que dé respuesta acertada a tareas concretas y trabaje en equipo; mientras que en el área de la docencia se persiguen los mismos objetivos pero que sus saberes sean para toda la vida. No obstante, en la labor docente, en el diseño instruccional de los cursos del currículo se vinculan ambas; fundamentalmente, en el perfil del egresado, puesto que, al terminar su labor de estudiante, el profesionista debe ser competente para responder a las demandas de su entorno laboral o empresarial. Ahí está la relación entre ellas.

Esta corriente formativa cobra tanta fuerza que puede constatarse internacionalmente, como en el caso de los proyectos Tuning en Europa y en Latinoamérica, así como Reflex y Profex en ambos continentes (López, 2013, p. 36).

Es sabido que para los constructivistas las competencias se adquieren a partir del conocimiento que tiene el individuo de su propia experiencia, su entorno. A ello se le suma la información que recibe del exterior, cómo la procesa, la analiza, la coteja, la reforma y descifra para formar parte de su conocimiento, sobre todo en contacto e interacción con los demás. Este es un proceso individual.

A partir de lo anterior se puede inferir que se ha formulado una gran diversidad de definiciones respecto al concepto de competencias, incluidas la de Roegiers y Perrenoud; sin embargo, para este estudio se tomó la que se ofrece en Proyecto Tuning (2006, citado en López, 2013), a saber: "Un conjunto de capacidades cognitivas y metacognitivas, intelectuales y prácticas, así como de valores éticos” (p. 40), ya que es la base de la filosofía de la Unacar. 


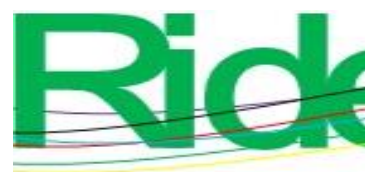

Revista Iberoamericana para la Investigación y el Desarrollo Educativo ISSN $2007-7467$

En este tenor, la clasificación de las competencias que se ofrece son dos: competencias genéricas y competencias específicas. Según López (2013): “A las competencias genéricas se les considera generadoras, en gran parte, del proceso de formación integral de los alumnos; también reciben el nombre de competencias transversales” (p. 41). Para este autor, son las competencias básicas, generales, que se involucran en los conocimientos transferibles. Y dentro de ellas está la competencia del dominio de las TIC, también estipulada en el Modelo Educativo Acalán de la Unacar (2017), el cual plantea el objetivo de que los estudiantes utilicen "adecuadamente las tecnologías de información y comunicación para acceder y generar información de manera efectiva y eficiente en el desempeño personal y profesional” (p. 74).

\section{Desarrollo}

El empleo de las TIC desde los primeros semestres en la universidad, en la modalidad presencial, tiene el objeto de propiciar una formación de mayor calidad en la educación del estudiante que le será útil para impulsar el conocimiento tecnológico del país; así, desde temprano en su carrera, se apropiará de las herramientas necesarias para los semestres siguientes; estará entrenado y competente en la utilización de las TIC, tal y como se espera de un profesional actual. Al respecto, García, Reyes y Godínez (2017) manifiestan lo siguiente:

No podemos dejar pasar inadvertido que la educación del futuro implicará un proceso de enseñanza-aprendizaje con ciertas particularidades como es el hecho de que podrá realizarse en cualquier instante, podrá ejecutarse en cualquier lugar y el ritmo de aprendizaje será personalizado (p. 7).

Por ello, profesores de la universidad, paralelamente a sus cursos presenciales, ponen en práctica las herramientas tecnológicas a su alcance, como puede ser el empleo de un aula virtual de aprendizaje para fortalecer los conocimientos y habilidades en un tema específico o materia, así como apoyar las destrezas de las competencias tecnológicas. En el NMC Horizon Report: Edición Educación Superior 2016 (Johnson et al., 2016), ya se apuntaba cómo la tecnología debía imperar en los espacios educativos y fuera de estos: plataformas en línea para un aprendizaje en la escuela y en la casa para reforzar los aprendizajes de los estudiantes. 


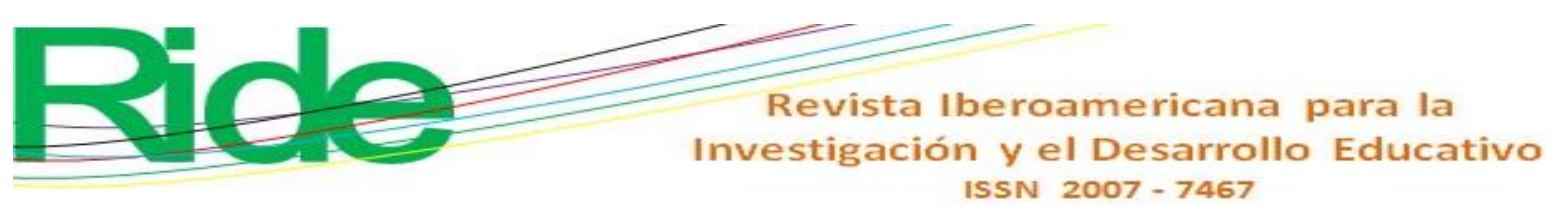

\section{Justificación}

En la actualidad, en diferentes universidades, los alumnos se enfrentan a problemáticas sociales, culturales y familiares que no les permiten mantener una estancia total en los centros de educación superior (Universidad Politécnica de Valencia/Instituto de Ciencias de la Educación/Área de Sistemas de Información y Comunicaciones, 2015) y demandan una docencia menos rígida que la presencial. Asimismo, los docentes han detectado insuficiencias en el aprendizaje o la práctica de determinados contenidos básicos para el nivel educativo en que el estudiante se encuentra, y recurren a otra modalidad para desarrollar competencias, entre ellas la tecnológica.

Algunas de las formas en las que se ha incursionado es el denominado blended learning o aprendizaje combinado. Sobre el tema, Abarca (2015) plantea que esta modalidad “combina la clase presencial con el uso de herramientas, tales como la instrucción interactiva, basada en la Web (...), los foros de discusión electrónicos, los contenidos para el trabajo autónomo y colaborativo" (p. 336).

Debido a esto, y tomando en cuenta el desarrollo de la tecnología educacional, además de la cantidad de alumnos a los que se enfrenta la educación superior en los últimos años, Flores, González y Reyes (2014) señalan que la cantidad de "programas educativos ofrecidos en una modalidad a distancia se encuentra en constante crecimiento, derivado de la oportunidad que brindan para que los estudiantes puedan organizar sus tiempos y forma de trabajo" (p. 1). Lo anterior quiere decir que, a partir de los esfuerzos de los docentes en involucrar las nuevas tecnologías en los procesos educativos, se logrará un profesional adaptado a los nuevos retos de la sociedad y su profesión: trabajo en equipo, habilidades y destrezas tecnológicas y, sobre todo, adaptabilidad al medio laboral actual.

\section{Planteamiento del problema}

A pesar de las aseveraciones anteriores, el entorno de la educación universitaria en el sureste mexicano dista mucho de la realidad que ofrece el centro y el norte del país. Según la Encuesta Nacional sobre Disponibilidad y Uso de Tecnologías de la Información en los Hogares [Endutih] (Instituto Nacional de Estadística y Geografía [Inegi], 2018) del año 2018, en el caso específico del estado de Campeche, en donde se sitúa el presente estudio, solo $51.3 \%$ de la población dispone de conexión a internet, tal y como se muestra en la tabla 1. 


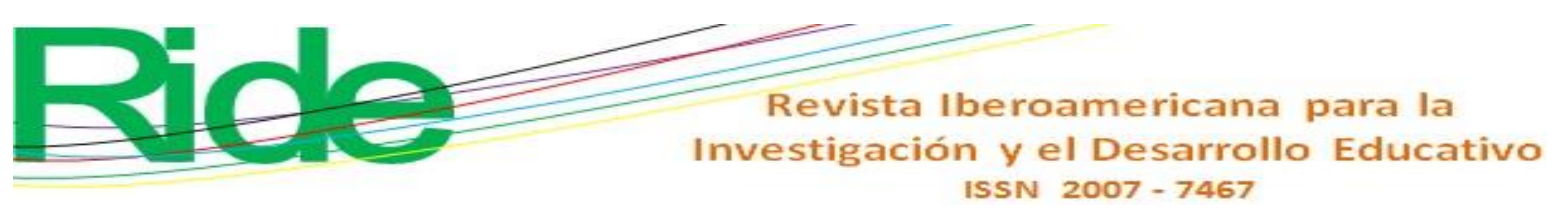

Tabla 1. Conexión a internet según la Endutih de 2018

\begin{tabular}{|c|c|c|c|c|c|c|}
\hline \multicolumn{7}{|c|}{$\begin{array}{l}\text { Hogares que disponen de conexión a internet por entidad federativa del sureste } \\
\text { mexicano en áreas urbano, rural en } 2018\end{array}$} \\
\hline \multirow[t]{3}{*}{ Entidad federativa } & \multicolumn{6}{|c|}{ Total } \\
\hline & \multicolumn{2}{|c|}{ Total } & \multicolumn{2}{|c|}{ Sí disponen } & \multicolumn{2}{|c|}{ No disponen } \\
\hline & Absolutos & $\%$ & Absoluto & $\%$ & Absoluto & $\%$ \\
\hline Campeche & 256284 & 100.0 & 131482 & 51.3 & 124802 & 48.7 \\
\hline Chiapas & 1360207 & 100.0 & 334629 & 24.6 & 1025578 & 75.4 \\
\hline Guerrero & 992229 & 100.0 & 347686 & 35.0 & 644543 & 65.0 \\
\hline Oaxaca & 1148216 & 100.0 & 338277 & 29.5 & 809939 & 70.5 \\
\hline Puebla & 1653961 & 100.0 & 655651 & 39.6 & 998310 & 60.4 \\
\hline Quintana Roo & 511117 & 100.0 & 379062 & 74.2 & 132055 & 25.8 \\
\hline Tabasco & 673068 & 100.0 & 301272 & 44.8 & 371796 & 55.2 \\
\hline Veracruz & 2481145 & 100.0 & 877589 & 35.4 & 1603556 & 64.6 \\
\hline Yucatán & 599396 & & 302363 & 50.4 & 297033 & 49.6 \\
\hline
\end{tabular}

Fuente: Inegi (2018)

A lo anterior se suman los datos que ofrece la misma encuesta nacional acerca de la cantidad de hogares que disponen de computadoras: solo $44.2 \%$ de los hogares en el estado de Campeche disponen de un equipo tecnológico. Por ende, la brecha digital en este estado es alarmante, sobre todo si se toma en cuenta que la educación apuesta a las competencias en las TIC. Esta información se visualiza en la tabla 2. 


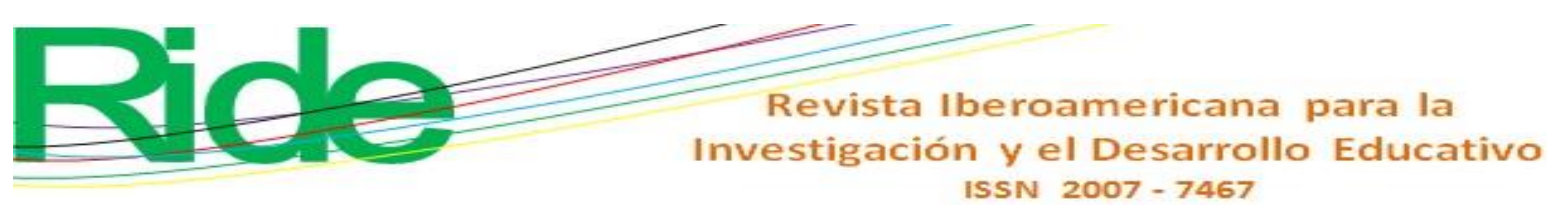

Tabla 2. Disposición de computadora según la Endutih del 2018

\begin{tabular}{|c|c|c|c|c|c|c|}
\hline \multicolumn{7}{|c|}{ Hogares que disponen de computadoras por entidad federativa del sureste } \\
mexicano en áreas urbano, rural en 2018 \\
\hline Entidad federativa & \multicolumn{5}{c|}{ Total } \\
\cline { 2 - 7 } & \multicolumn{2}{|c|}{ Total } & \multicolumn{2}{c|}{ Sí disponen } & \multicolumn{2}{c|}{ No disponen } \\
\cline { 2 - 7 } & Absolutos & $\%$ & Absoluto & $\%$ & Absoluto & $\%$ \\
\hline Estados Unidos & 34699 & 100.0 & 1557418 & 44.9 & 19125 & 55.1 \\
Mexicanos & 617 & & 2 & & 435 & \\
\hline Campeche & 256284 & 100.0 & 113344 & 44.2 & 142940 & 55.8 \\
\hline Chiapas & 1360207 & 100.0 & 328258 & 24.1 & 1031949 & 75.9 \\
\hline Guerrero & 992229 & 100.0 & 294989 & 27.7 & 697240 & 70.3 \\
\hline Oaxaca & 1148216 & 100.0 & 356756 & 31.1 & 791460 & 68.9 \\
\hline Puebla & 1653961 & 100.0 & 610823 & 36.9 & 1043138 & 63.1 \\
\hline Quintana Roo & 511117 & 100.0 & 245572 & 48.0 & 265545 & 52.0 \\
\hline Tabasco & 673068 & 100.0 & 233183 & 34.6 & 439885 & 65.4 \\
\hline Veracruz & 2481145 & 100.0 & 761686 & 30.7 & 1719459 & 69.3 \\
\hline Yucatán & 599396 & & 285137 & 47.6 & 314259 & 52.4 \\
\hline & & & & & & \\
\hline
\end{tabular}

Nota: Proporciones respecto del total de hogares. Cifras preliminares. Cifras correspondiente al mes de mayo.

Fuente: Inegi (2018)

Por todo lo expuesto anteriormente, antes de iniciar un curso, un taller para la docencia o la investigación con el empleo de algún programa o herramienta tecnológica, surgen las preguntas de investigación: ¿qué habilidades y destrezas tecnológicas tienen los estudiantes de los primeros semestres de la Unacar ante las realidades de su entorno social y familiar?, ¿han tenido experiencias en los niveles educativos precedentes que hayan desarrollado su nivel de competencia en las TIC y el trabajo colaborativo? 


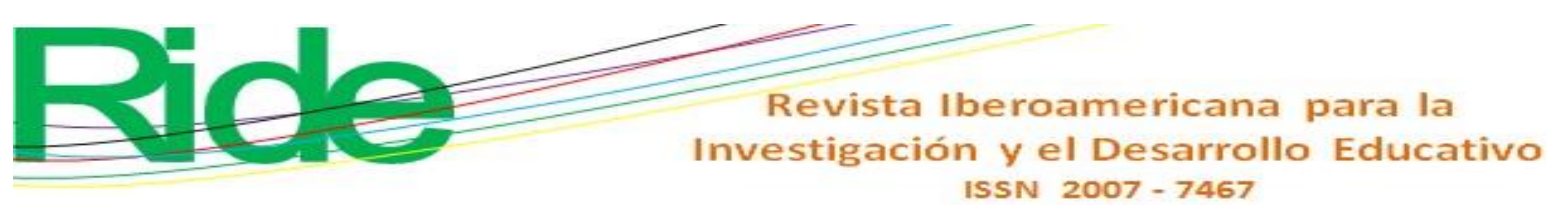

\section{Objetivo general}

Determinar qué tipo de competencia en el empleo de las TIC presentan los estudiantes de los primeros semestres de la licenciatura en Educación perteneciente a la Facultad de Ciencias Educativas de la Unacar como futuros profesionales en cuyos manos recaerá la noble tarea de formar a los especialistas de diferentes áreas en México.

\section{Método}

Se determinó realizar un diagnóstico acerca del conocimiento y la práctica, las habilidades desarrolladas en el empleo de la tecnología, así como las condiciones personales que poseen los estudiantes de los primeros semestres de la licenciatura en Educación de la Facultad de Ciencias Educativas de la Universidad Autónoma del Carmen, con miras a enfrentar cursos en línea o semipresenciales que apoyen las materias que reciben de forma presencial, debido a los nuevos roles que deben desempeñar los docentes y los estudiantes en el siglo XXI con el empleo de las TIC.

Se realizó una investigación de tipo cuantitativa, con un alcance descriptivo, puesto que, como enuncia Hernández, Fernández y Baptista (2010), "busca especificar propiedades, características, rasgos importantes de cualquier fenómeno que se analice. Describe tendencias de un grupo o población" (p. 80); en este caso, acerca del perfil del estudiante desde el punto de vista de su competencia tecnológica.

Cabe señalar que aquí se entiende por perfil de usuario el conjunto de características o preferencias que la persona tiene sobre sus búsquedas de Internet o en los sitios web que frecuenta (Cortés y De la Cruz, 2016). Son las habilidades que presenta el estudiante en el manejo de la tecnología para desempeñarse en otra modalidad que no sea la presencial.

\section{Muestra}

Para este estudio la muestra poblacional constó de un total de 52 estudiantes, los cuales conforman los dos grupos de primer semestre, en el ciclo escolar de febrero a julio de 2019, de la licenciatura en Educación ya especificada, quienes tienen un perfil similar en cuanto a edad, sexo y escuelas de procedencia.

Se empleó una muestra no aleatoria, ya que los grupos están integrados de manera natural o normal para la actividad que fueron creados; no hay una asignación aleatoria (Bono, 


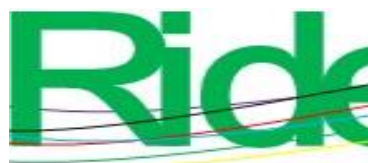

Revista Iberoamericana para la Investigación y el Desarrollo Educativo ISSN $2007-7467$

2012). Esto sucede en los salones de clases, en los colegios u otros centros. Por ello, para determinar el perfil del usuario en cuanto a la competencia y las habilidades de uso en la tecnología, participaron todos los estudiantes como población delimitada.

\section{Técnicas empleadas para la recogida de información}

El instrumento que se utilizó fue una encuesta de opción múltiple basada en un cuestionario de tipo diagnóstico, con el objetivo de conocer la competencia tecnológica del estudiante: qué experiencia ha tenido en el uso de una plataforma educativa, qué condiciones materiales personales tiene en su casa y la escuela para participar en el curso, qué herramientas tecnológicas domina, entre otros aspectos de interés para este tema, como se podrá constatar en el análisis de los resultados.

Para ello, se consultó la encuesta titulada Cuestionario sobre usos de las TIC entre estudiantes universitarios barineses (Universitat Oberta de Catalunya, 2006), y se adaptó a las características de los estudiantes de la Unacar, población objeto de estudio, las condiciones particulares de las universidades en México, así como la zona en la que se realiza este estudio. Este cuestionario fue revisado y validado por el cuerpo académico Unacar CA35, cuya línea de investigación es la siguiente: "Tecnologías de la información y las comunicaciones para la colaboración de la enseñanza y el aprendizaje”.

Esta encuesta se empleó cara a cara (Batthyány y Cabrera, 2011), gracias a lo cual se pudo tener contacto directo con el estudiante mientras contestaba las preguntas, con la finalidad de precisar términos o dudas que pudieran surgir; para ello, de forma personal, se aplicó el cuestionario en físico integrado por 20 ítems.

Anteriormente, este instrumento fue aplicado a un grupo piloto, $\mathrm{y}$, de acuerdo con el alfa de Cronbach, obtuvo una fiabilidad alta, con un valor de 0.92.

Los resultados se analizaron de forma estadística a partir de la herramienta del programa de Excel con la intención de obtener las frecuencias acerca de los valores obtenidos para las respuestas a las interrogantes. 
Resultados

En los resultados presentados en este texto se tuvieron en cuenta las respuestas que podían impactar en la búsqueda de la competencia tecnológica de los estudiantes, así como las condiciones que poseen de forma personal, por lo que no se tomaron algunos ítems cuyos resultados dependían de una respuesta anterior que fue negativa.

La figura 1 permite ver gráficamente cómo está distribuida la muestra de este estudio en cuanto a género: $71 \%$ femenino y $29 \%$ de masculino.

Figura 1. Distribución de género de la muestra objeto de estudio

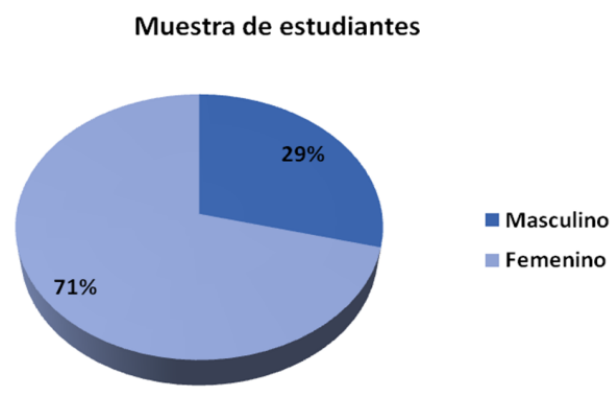

Fuente: Elaboración propia

La pregunta dos tuvo el objetivo de conocer el rango de edad de los estudiantes. Las respuestas oscilan entre los 18 y 24 años, tal y como se aprecia en la figura 2.

Figura 2. Distribución de la edad de la muestra Edad de los estudiantes

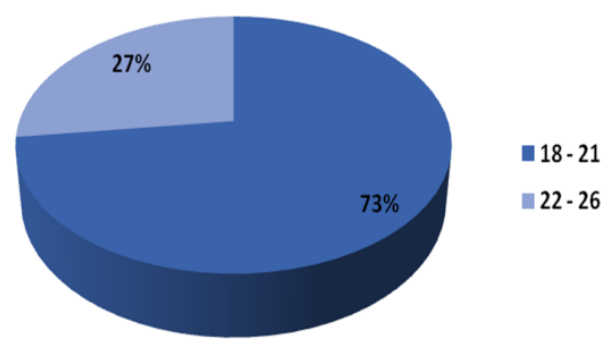

Fuente: Elaboración propia

En la pregunta tres se indagó sobre cuántas horas navegan en la Red los participantes. Los resultados indican que, del total, la mayor parte de ellos, $44 \%$, navega en Internet por un periodo de 13 a 20 horas semanales (ver figura 3). 


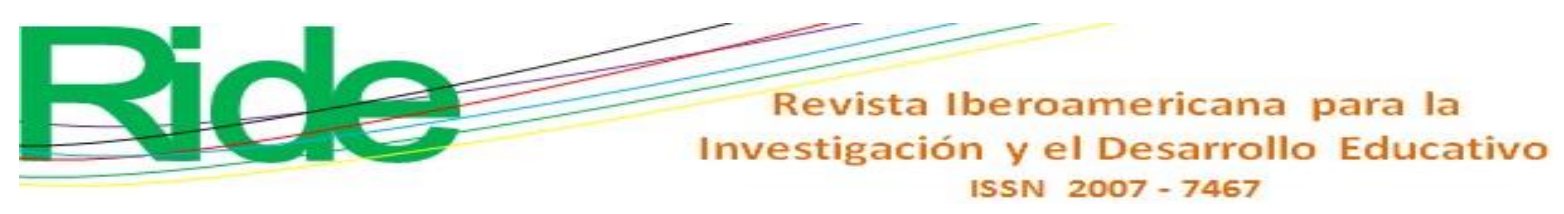

Figura 3. Horas de navegación en Internet a la semana

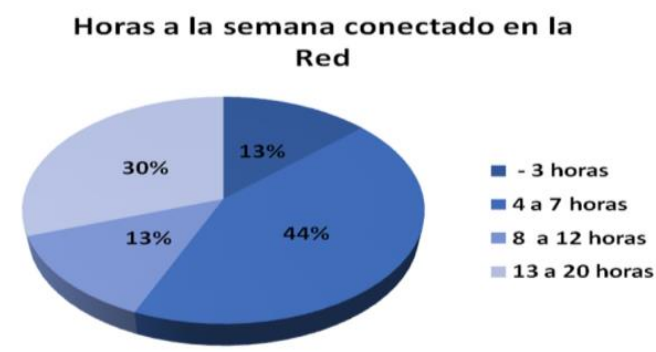

Fuente: Elaboración propia

Al respecto, se considera que no es suficiente el tiempo si pensamos que deben realizar actividades individuales, en conjunto, colaborativas, o leer información para el trabajo de sus clases y proyectos.

En la pregunta cuatro, donde se indaga si posee equipo personal de cómputo el encuestado, se evidencia que muchos de los estudiantes no lo tienen. De hecho, la mitad de estos no posee computadora, laptop o tableta ni teléfono inteligente, como se puede apreciar en la figura 4.

Figura 4. Estudiantes con equipo personal de cómputo

Posee equipo electrónico personal

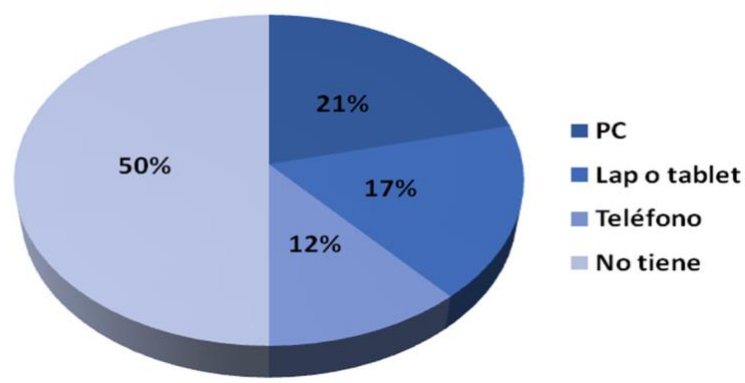

Fuente: Elaboración propia

Estos datos se relacionan estrechamente con el hecho de que, en la mayoría de los casos, los alumnos encuestados no tienen servicio de internet en sus casas, tal como lo dejan ver las respuestas a la interrogante cinco, cuando se indaga si cuentan con algún proveedor de internet en sus casas (ver figura 5). 


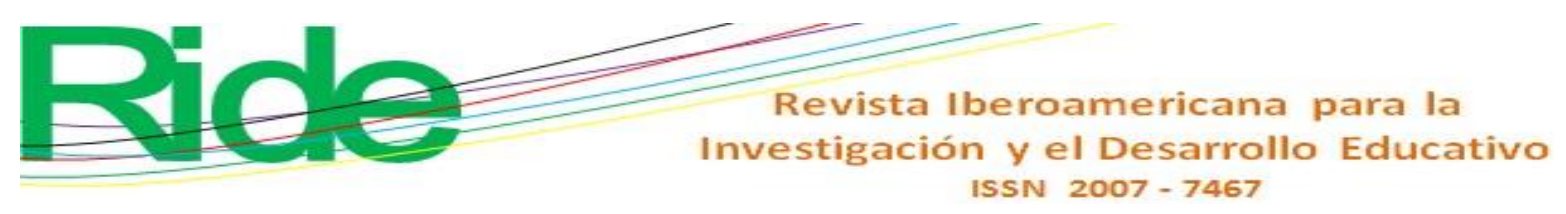

Figura 9. Cuentas de correo electrónico que posee

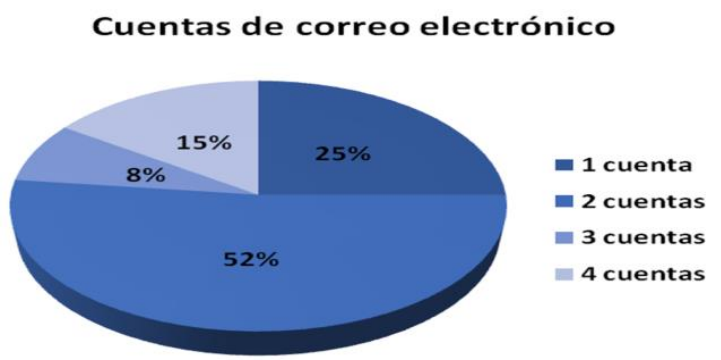

Fuente: Elaboración propia

Esta información coincide con los datos arrojados por Cázares y Urbiola (2014): "Facebook obtuvo un 23.7 \% de preferencia y la opción de correo electrónico un 73.1 \%" (p. 21), lo que permite analizar que los estudiantes se mantienen comunicados por medio del correo electrónico, aunque actualmente hay otras vías, como el WhatsApp, Skype, Facebook; no obstante, como se decía, esta sigue siendo la vía esencial para aquellos que no tienen teléfonos inteligentes, equipos personales y servicio de internet en sus casas.

En el ítem 12, los encuestados debían contestar si utilizan la computadora u otra herramienta tecnológica para realizar sus presentaciones en las clases: solo $19 \%$ respondió que lo hace frecuentemente, mientras que la mayoría solo a veces, como se aprecia en la figura 10. Estas presentaciones son diapositivas, buscar imágenes o tomar textos de Internet.

Figura 10. Empleo de las TIC para realizar presentaciones en las clases

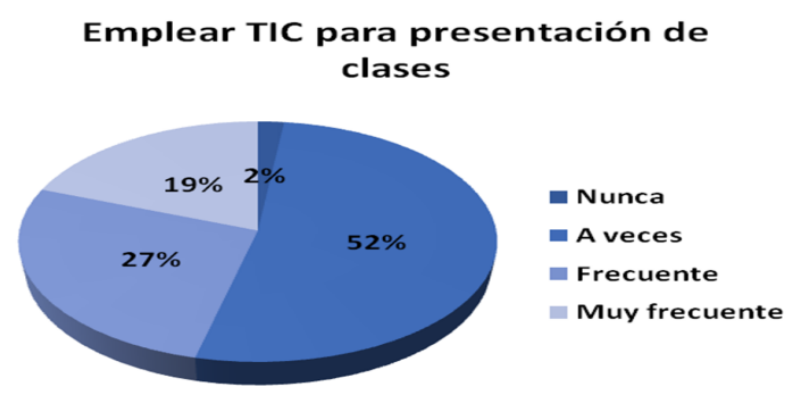

Fuente: Elaboración propia

La respuesta a esta pregunta refuerza la idea del poco uso del Internet, puesto que, al cuestionarles en la pregunta 13 si han establecido comunicación online con sus compañeros para realizar alguna actividad académica, los datos indican que solo $17 \%$ lo ha hecho de forma muy frecuente, y $37 \%$ de forma frecuente, como se expresa en la figura 11 . Pocos son los estudiantes encuestados que se han enfrentado a actividades colaborativas. 


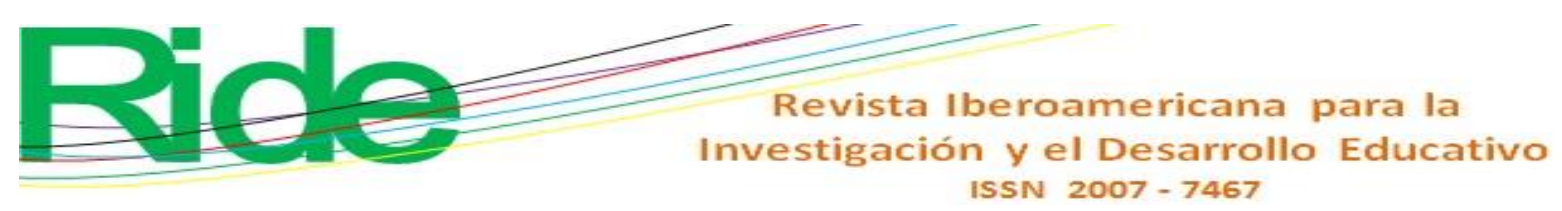

Figura 14. Experiencia en el uso de programas informáticos

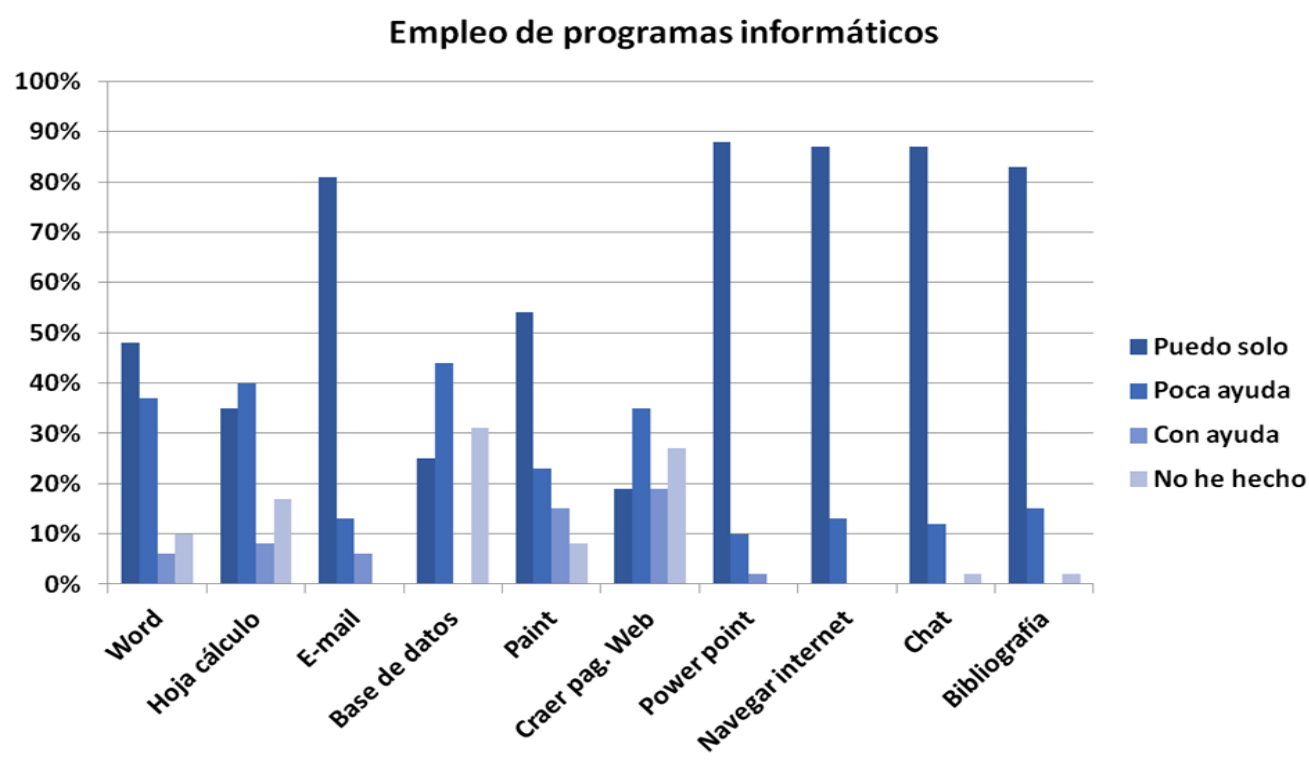

Fuente: Elaboración propia

Los escenarios en los que los estudiantes han aprendido a emplear estas herramientas son diversos, por lo que en la pregunta 18 se solicita que marque una opción que identifique dónde ha obtenido sus actuales habilidades y conocimientos sobre las TIC. En la figura 15 se muestran los resultados al respecto: $46 \%$ indica que en el nivel medio superior, $25 \%$ con amigos y otro $29 \%$ a través de tutoriales. Esto quiere decir que cada vez son más variados los espacios de aprendizaje, si tomamos en consideración el porcentaje de estudiantes que no tienen internet ni equipos personales de cómputo en la actualidad.

Figura 15. Lugares en los que obtuvo habilidades y conocimientos sobre TIC

\section{Conocimientos y habilidades en el uso de TIC}

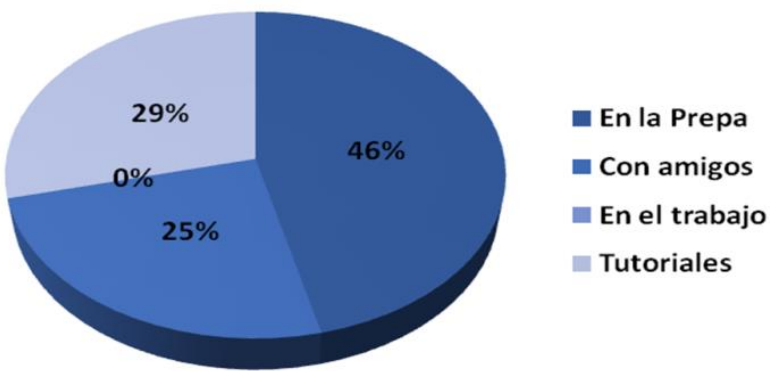

Fuente: Elaboración propia 


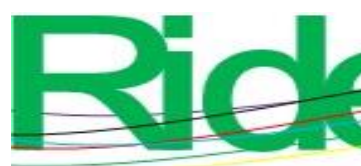

Es de particular interés para este estudio la pregunta 20, la cual indaga sobre si en sus estudios preparatorianos tuvieron la oportunidad de interactuar con algunas herramientas de e-learning, y se refleja que casi la mitad de los estudiantes, $49 \%$, ha incursionado en páginas de Internet, $17 \%$ ha participado en foros, $21 \%$ ha trabajado en un aula virtual de aprendizaje y $13 \%$ ha establecido debates en línea, como se aprecia en la figura 16; todo lo cual coincide con los datos que han aportado los estudiantes en las preguntas anteriores: menos de la mitad de los encuestados tiene desarrollada su competencia en el empleo de las TIC.

Figura 16. Incursión en otras herramientas de la Web Empleo de otras herramientas

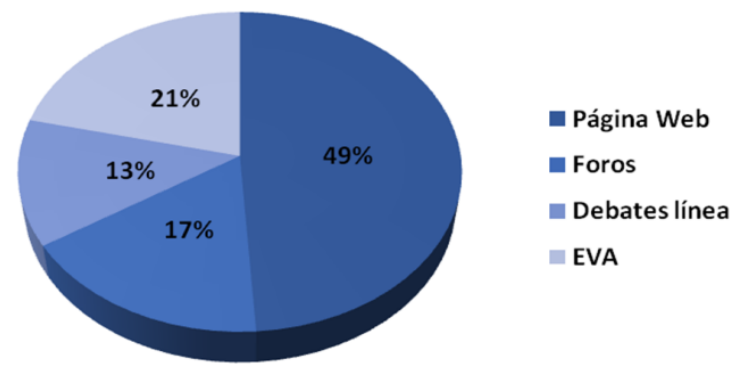

Fuente: Elaboración propia

De todo lo anterior, se puede plantear que aproximadamente $50 \%$ de los usuarios a quienes va dirigido este proyecto no posee un equipo de cómputo personal, tampoco puede contar con internet en su casa, ha tenido poca experiencia en el empleo de recursos de la Web para el trabajo individual y colectivo y no ha desarrollado destrezas en la aplicación de algunas herramientas tecnológicas necesarias para el nivel educativo que cursa.

A pesar de todas las limitaciones e inconvenientes que han presentado estos estudiantes en su competencia acerca del empleo de las TIC, en la pregunta 19 se cuestiona sobre la importancia que consideran tienen las TIC para su futura profesión. Como se puede ver en la figura $17,52 \%$ considera que es muy importante este conocimiento y $40 \%$ lo considera importante; solo $8 \%$ considera de cierta importancia. Pensamos que estos son los que están más alejados de los recursos tecnológicos en su vida diaria, o que aún no están convencidos del cambio del paradigma del futuro docente, porque están en los primeros semestres en la universidad. 


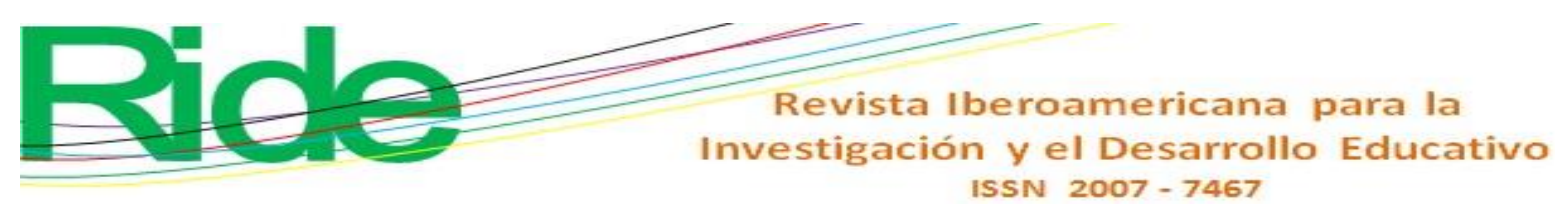

Figura 17. Importancia de las TIC en tu futura profesión

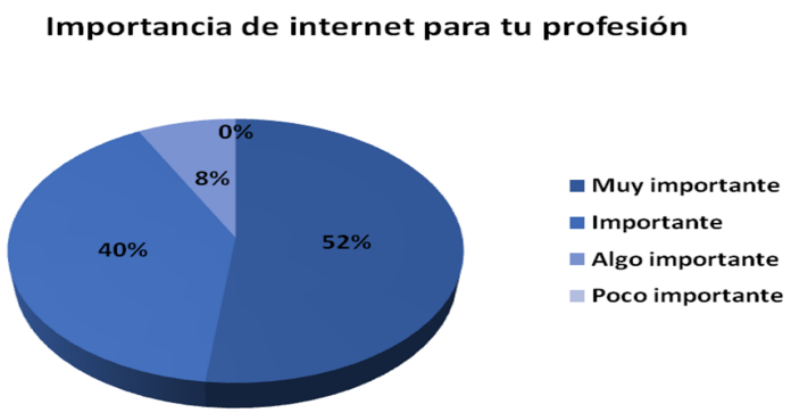

Fuente: Elaboración propia

\section{Discusión}

De todo lo presentado anteriormente, se puede resumir que aproximadamente $50 \%$ de la muestra de estudio no posee un equipo de cómputo personal, no cuenta con servicio de internet en su casa y ha tenido poca experiencia en el empleo de recursos de la Web para el trabajo individual y colectivo. Asimismo, no ha desarrollado destrezas en la aplicación de algunas herramientas tecnológicas necesarias para el nivel educativo que cursa ni tampoco para desenvolverse en el mundo civilizado actual, a pesar de que reconoce su importancia para su profesión.

$\mathrm{Al}$ respecto, la Organización de las Naciones Unidas para la Educación, la Ciencia y la Cultura [Unesco] (1996, citada en Posada, 2015) plantea:

Esta revolución tecnológica constituye a todas luces un elemento esencial para entender nuestra sociedad, en la medida que crea nuevas formas de socialización, e incluso nuevas definiciones de identidad individual y colectiva (...). El conocimiento es, justo con las TIC el elemento central de nuestra actual estructura civilizatoria (p. 193).

En el manifiesto de la Unesco se deja en claro la necesidad del empleo de las tecnologías para la vida y el desarrollo de la sociedad; por ende, la responsabilidad de las universidades en el sureste ante este desafío es esencial.

Este estudio es una muestra de lo que sucede en los primeros semestres de la licenciatura en Educación de la Facultad de Ciencias Educativas de la Unacar. Sin duda la muestra es pequeña; sin embargo, la incompetencia tecnológica de los estudiantes es una realidad. Este acercamiento resultará aún más interesante si la población se amplía a 


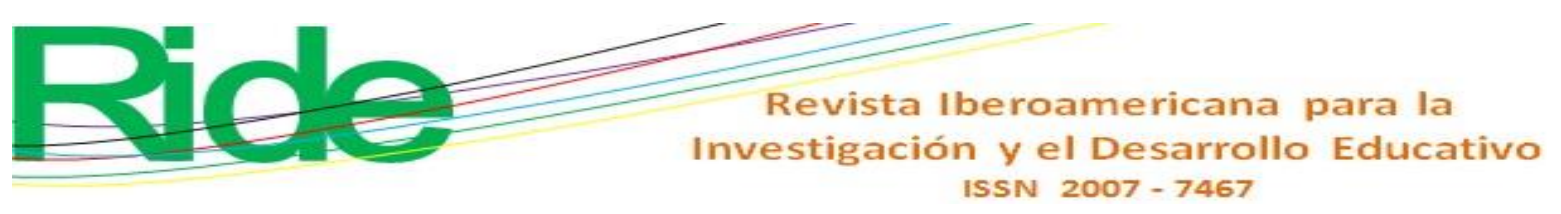

otras especialidades, y se abarcan otras universidades del sureste. Estamos seguros de que el perfil del usuario en tecnología es similar.

Se puede resumir que, a partir de los resultados observados, los estudiantes de la Lic. en Educación de la Unacar están conectados a la Web de forma pasiva: prefieren recibir la información y no trabajar con ella para lograr productos o información porque, casi en su totalidad, no saben cómo lograrlo; y, por la misma razón, tampoco logran interactuar con otros compañeros. En suma, debido a su incompetencia tecnológica, no pueden desempeñar un rol más activo. De las estrategias que se deriven de estos resultados dependerá un cambio en el empleo de las TIC de dichos estudiantes.

\section{Conclusiones}

Como respuesta a los resultados obtenidos en este estudio, las instituciones de educación superior en el sureste mexicano deben tener un compromiso con el desarrollo de esta competencia, ya que si los docentes actuales de la Unacar se plantean la intención de crear cursos en aulas virtuales de aprendizaje y utilizar herramientas para la investigación con sus estudiantes de la Facultad de Ciencias Educativas tendrían un gran obstáculo a superar.

De la misma forma, deberán establecerse estrategias a corto, mediano y largo plazos para que los estudiantes sientan el interés en un nuevo aprendizaje mediado por la tecnología.

A corto plazo, una de las medidas para paliar las dificultades en cuanto a la disponibilidad de recursos es crear nuevos espacios físicos para que los estudiantes con mayor brecha digital tengan acceso desde la institución a las herramientas tecnológicas y el Internet.

A mediano plazo, será necesario capacitar a los docentes que, de una forma u otra, han quedado rezagados en estos campos, ya sea por la diferencia generacional o desidia, puesto que ser educador implica una responsabilidad insoslayable con la sociedad y la enseñanza.

Ello implica una nueva forma de enseñar, una nueva forma de interactuar en el salón de clases y fuera de este, por lo que los estudiantes y docentes de la Facultad de Ciencias Educativas de la Unacar deben repensar su labor actuar como propiciadores de cambios. 


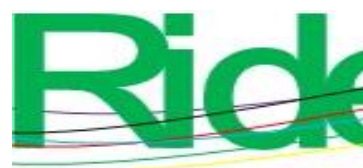

Revista Iberoamericana para la Investigación y el Desarrollo Educativo ISSN $2007-7467$

Y a largo plazo, los modelos y diseños curriculares en los centros de educación superior cuya modalidad sea presencial deberán incorporar cursos y talleres extracurriculares en la modalidad a distancia o semipresencial como apoyo a las materias que reciben los estudiantes, con el objetivo de eliminar la brecha digital que existe y en apoyo a la competencia tecnológica que debe imperar en la educación del siglo XXI.

\section{Referencias}

Abarca, Y. (2015). El uso de las TIC en la educación universitaria: motivación que incide en su uso y frecuencia. Revista Lenguas Modernas, (22), 335-349. Recuperado de https://revistas.ucr.ac.cr/index.php/rlm/article/view/19692.

Arras, A., Torres, C. A. y García, A. (2011). Competencias en Tecnologías de Información y Comunicación (TIC) de los estudiantes universitarios. Revista Latina de Comunicación Social, (66), 130-152. Recuperado de http://www.revistalatinacs.org/11/art/927_Mexico/06_Arras.html.

Batthyány, K. y M. Cabrera (2011). Metodología de la investigación en Ciencias Sociales. Apuntes para un curso inicial. Montevideo: Departamento de Publicaciones, Unidad de Comunicación de la Universidad de la República (UCUR)

Cázares, I. y Urbiola, A. (2015). Estudiantes universitarios, en el uso de las herramientas tecnológicas ¿activos o pasivos? Revista Educación y Humanismo, 17(28), 15-28. Recuperado de http://dx.doi.org/10.17081/eduhum.17.28.1163.

Cortés, P. N. y De la Cruz, C. (2016). El perfil del usuario. Boletín UPIITA, (39). Recuperado de http://www.boletin.upiita.ipn.mx/index.php/ciencia/510-cyt-numero-39/338-elperfil-de-usuario.

Flores, G. I., González, A. y Reyes, J. L. (2014). El Paradigma del Constructivismo en la Educación a Distancia. TEPEXI Boletín Científico de la Escuela Superior Tepeji del Río, 1(2). Recuperado de https://repository.uaeh.edu.mx/revistas/index.php/tepexi/article/download/1506/491 4? inline $=1$.

García, M. R., Reyes, J. y Godínez, G. (2017). Las Tic en la educación superior, innovaciones y retos. Revista Iberoamericana de las Ciencias Sociales y Humanísticas, 6(12). Recuperado de https://dialnet.unirioja.es/servlet/articulo?codigo=6255413. 


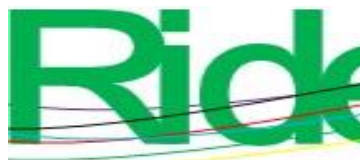

Revista Iberoamericana para la Investigación y el Desarrollo Educativo ISSN $2007-7467$

Hernández, R., Fernández, C y Baptista, P. (2010). Metodología de la investigación (5.a ed.). México: McGraw-Hill.

Instituto Nacional de Estadística y Geografía [Inegi]. (2018). Encuesta Nacional sobre Disponibilidad y Uso de Tecnologías de la Información en los Hogares (Endutih). México: Instituto Nacional de Estadística y Geografía. Recuperado de https://www.inegi.org.mx/programas/dutih/2018/.

Johnson, L., Adams, S., Cummins, M., Estrada, V., Freeman, A. y Hall, C. (2016). NMC Informe Horizon 2016 Edición Superior de Educación. Austin, Estados Unidos: The New Media Consortium.

López, M. A. (2013). Aprendizajes, competencias y TIC. México: Pearson

Posada. A. (2015). Nuevos comportamientos de los jóvenes universitarios frente a la comunicación mediados por las TIC. Revista Itinerario Educativo, 29(66), 189-203.

Universidad Autónoma del Carmen [Unacar]. (2017). Modelo Educativo Acalán. Campeche, México: Universidad Autónoma del Carmen. Recuperado de http://www.unacar.mx/Dir_General_Academica/Documentos/Modelo_Educativo_A calan/Modelo_Ed_Acalan2017.pdf.

Universitat Oberta de Catalunya. (2006). Cuestionario sobre usos de las TIC entre estudiantes universitarios barineses. Barcelona, España: Universitat Oberta de Catalunya.

Universidad Politécnica de Valencia/Instituto de Ciencias de la Educación/Área de Sistemas de Información y Comunicaciones. (2015). Plan de Acciones para la Convergencia Europea (PACE) Los objetos de aprendizaje como recurso para la docencia universitaria: criterios para su elaboración. España: Universidad Politécnica de Valencia/Instituto de Ciencias de la Educación/Área de Sistemas de Información y Comunicaciones. Recuperado de www.aqu.cat/doc/doc_22391979_1.pdf. 


\begin{tabular}{|c|c|}
\hline & $\begin{array}{l}\text { Revista Iberoamericana para la } \\
\text { Investigación y el Desarrollo Educativo } \\
\text { ISSN } 2007-7467\end{array}$ \\
\hline Rol de Contribución & Autor (es) \\
\hline Conceptualización & Gisela Aquilea Diez Irizar \\
\hline Metodología & $\begin{array}{l}\text { Gisela Aquilea Diez Irizar<igual>, Beariz Herrera } \\
\text { Sánchez<igual> }\end{array}$ \\
\hline Software & $\begin{array}{l}\text { Beariz Herrera Sánchez <igual>, Jesús Alejandro Flores } \\
\text { Hernández <igual> }\end{array}$ \\
\hline Validación & Gisela Aquilea Diez Irizar \\
\hline Análisis Formal & Gisela Aquilea Diez Irizar, Beariz Herrera Sánchez, <igual> \\
\hline Investigación & Gisela Aquilea Diez Irizar, Beariz Herrera Sánchez, <igual> \\
\hline Recursos & Gisela Aquilea Diez Irizar, Beariz Herrera Sánchez, <igual> \\
\hline Curación de datos & Gisela Aquilea Diez Irizar, Beariz Herrera Sánchez, <igual> \\
\hline $\begin{array}{l}\text { Escritura - Preparación del } \\
\text { borrador original }\end{array}$ & Gisela Aquilea Diez Irizar \\
\hline $\begin{array}{l}\text { Escritura - Revisión y } \\
\text { edición }\end{array}$ & $\begin{array}{l}\text { Gisela Aquilea Diez Irizar <principal>, Beariz Herrera Sánchez } \\
\text { <apoyo>, Jesús Alejandro Flores Hernández <apoyo> }\end{array}$ \\
\hline Visualización & Beariz Herrera Sánchez \\
\hline Supervisión & Beariz Herrera Sánchez \\
\hline $\begin{array}{l}\text { Administración de } \\
\text { Proyectos }\end{array}$ & Gisela Aquilea Diez Irizar, Beariz Herrera Sánchez <igual> \\
\hline Adquisición de fondos & Gisela Aquilea Diez Irizar, Beariz Herrera Sánchez <igual> \\
\hline
\end{tabular}

